\title{
Les poissons du bassin de la rivière Ulindi, à l'Est de la République Démocratique du Congo : revue de la littérature
}

\author{
Gabriel MUKABO OKITO ${ }^{1,2 *}$, Joseph MASIRIKA MATUNGURU ${ }^{1}$, \\ Leblanc HERI MUZUNGU ${ }^{3}$, Papy LUBUNGA DUNI ${ }^{4}$, Anicet SINDAYIHEBURA ${ }^{2}$, \\ Claver SIBOMANA ${ }^{2}$, Venant NSHOMBO MUDERHWA ${ }^{3}$, Jean-Claude MICHA ${ }^{5}$ et \\ Gaspard NTAKIMAZI ${ }^{2}$
}

\author{
${ }^{1}$ Ecole Doctorale de l'Université du Burundi, Facultés des Sciences, Laboratoire de Biodiversité, Ecologie et \\ Environnement. B.P. 2700 Bujumbura-Burundi. \\ ${ }^{2}$ Section Eaux et Forêts, Institut Supérieur d'Agrovétérinaire et de Conservation de la nature de Minembwe, \\ (ISAVC/Minembwe). BP 6295 Bujumbura-Burundi. \\ ${ }^{3}$ Département de Biologie, Université Officielle de Bukavu (U.O.B./Bukavu). BP 570 Bukavu-RD Congo. \\ ${ }^{4}$ Département de Biologie du Centre de Recherche en Hydrobiologie d'Uvira (CRH-Uvira). B.P. 73 Uvira, \\ $R D$ Congo. \\ ${ }^{5}$ Unité de Recherche en Biologie Environnementale et Evolutive, Département de Biologie, Université de \\ Namur. BP 5000 Namur, Belgique. \\ *Auteur correspondant ; E-mail : mgokito@ gmail.com ; Tél. +243821270424
}

\section{RÉSUMÉ}

Cette revue de la littérature sur les poissons du bassin hydrographique de la rivière Ulindi à l'Est de la République Démocratique du Congo a été réalisée dans le but d'avoir une idée sur l'état des connaissances actuelles de son ichtyofaune en vue de définir les orientations pour leurs études ultérieures dans cette région non encore prospectée en profondeur et dont la plupart des rivières se trouvent actuellement menacées par des activités anthropiques. Cette étude présente une vue globale des rivières du Bassin de la rivière Ulindi avec une liste systématique non exhaustive d'environ 31 espèces de poissons appartenant à 17 genres, 9 Familles et 4 ordres, basée sur la méta-analyse des différentes études taxonomiques de l'ichtyofaune du Bassin de la Rivière Ulindi, réalisées depuis l'époque coloniale du Congo-Belge jusqu'en 2019. Les résultats de cette étude constituent une aide-mémoire pour les services publics en charge de la gestion de l'environnement dans la gestion de la biodiversité ichtyologique de cet écosystème à intérêt socio-économique considérable dans la région, d'une part et une liste de contrôle aux scientifiques pour des études ultérieures.

(C) 2020 International Formulae Group. All rights reserved.

Mots clés : Ichtyofaune, Ulindi, République Démocratique du Congo, revue de la littérature, liste de contrôle. 


\title{
Fishes from the Ulindi river basin, East of the Democratic Republic of Congo: review of the literature
}

\begin{abstract}
This review of the literature on fish in the Ulindi river basin in the east of the Democratic Republic of Congo was carried out with the aim of gaining an idea of the state of current knowledge of its fish fauna in order to define guidelines for their further studies in this region not yet explored in depth and most of the rivers of which are currently threatened by anthropogenic activities. This study presents a global view of the rivers of the Ulindi river basin with a non-exhaustive systematic list of approximately 31 fish species belonging to 17 genera, 9 Families and 4 orders, based on the meta-analysis of the various taxonomic studies of the ichtyofauna of the Ulindi river basin, carried out since the colonial times of the Belgian Congo until 2019. The results of this study constitute a baseline for the public services in charge of environmental management in the management of the ichthyological biodiversity of this ecosystem with considerable socio-economic interest in the region, on the one hand and a checklist to scientists for further studies.
\end{abstract}

(C) 2020 International Formulae Group. All rights reserved.

Keywords: Ichtyofauna, Ulindi, Democratic Republic of Congo, literature review, checklist.

\section{INTRODUCTION}

Le bassin du Congo possède la plus grande diversité de poissons du continent africain et se classe au deuxième rang mondial pour la diversité des espèces, après le bassin de l'Amazone. Ces poissons constituent une ressource alimentaire importante pour les populations locales et, bien que le bassin du Congo soit un point chaud de biodiversité, la faune ichtyologique est à peine étudiée (Moelants, 2015; Harrison et al., 2016). Les quelques études menées dans la province ichtyologique du Congo ont été plus ciblées sur certaines rivières des cours inférieur et moyen (Banister et Bailey, 1979; Ibala et Masmoudi, 2010; Wamuini et al., 2010 ; Goudiaby et Diop, 2015; Danadu et al., 2016; Decru et al., 2017a; 2017b; Decru et al., 2017; Musschoot et Snoeks, 2017) . Pour le cours supérieur du bassin du Congo (Figure 1), auquel appartient la rivière Ulindi, la plupart des études qui traitent de la diversité et de l'écologie de l'ichtyofaune des affluents majeurs du fleuve Congo ne concernent que les bassins des rivières Elila, Lowa, Luhoho ( Pellegrin, 1935; Marlier, 1954; Kisekelwa et al., 2014: 2016; Okito et al., 2017).

La rivière Ulindi n'a pas encore fait l'objet d'étude sur la biodiversité ichtyologique depuis le départ des colons Belges. Et pourtant, c'est une rivière très importante dans la région qui traverse une grande région biogéographique et semble contenir une riche diversité ichtyologique jusque-là inconnue dans son ensemble. Elle est la plus longue rivière de la Province du SudKivu en République Démocratique du Congo et prend sa source à Niakunduku non loin du Lac Lungwe dans les hauts plateaux d'Itombwe à plus de $2800 \mathrm{~m}$ d'altitude (Marlier, 1954). Elle traverse les territoires de Walungu, Mwenga, et Shabunda au Sud-Kivu pour aller se jeter dans le fleuve Congo après avoir parcouru le territoire de Kalima et Punia en Province du Maniema. La rivière Ulindi joue également un rôle important dans la subsistance des populations environnantes. Outre les activités de pêche qui s'y organisent avec des méthodes et techniques souvent destructives (pêches aux explosifs, produits toxiques...), il se pratique également des activités minières avec plus de 175 dragues suceuses artisanales et 4 dragues industrielles à chaînes à godets (de la société chinoise « Kunhou Mining Group ») utilisant environ $358 \mathrm{~kg}$ de mercure par semaine pour l'amalgame de l'or (COSOC-GL 2015). Dès lors, certaines espèces piscicoles présentes 
dans cette partie du bassin du fleuve Congo risquent de disparaître avant qu'elles ne soient connues. Et pourtant, la connaissance de la biodiversité d'un milieu est une condition sine qua non pour sa gestion durable (Freyhof et al., 2015; Hermoso et al., 2016).

C'est dans le souci d'apporter une contribution sur la connaissance actuelle de la composition de l'ichtyofaune du bassin de la rivière Ulindi que nous avons mené cette recherche bibliographique afin d'obtenir une liste de ses poissons qui puisse nous servir de base pour les recherches futures.

Ainsi, dans les paragraphes qui suivent, nous présentons un aperçu sur la méthodologie utilisée dans le cadre de cette étude, les résultats de la recherche bibliographique (liste systématique des espèces de poissons connues à ce jour et leur répartition dans ce bassin), suivi d'une discussion et conclusion.

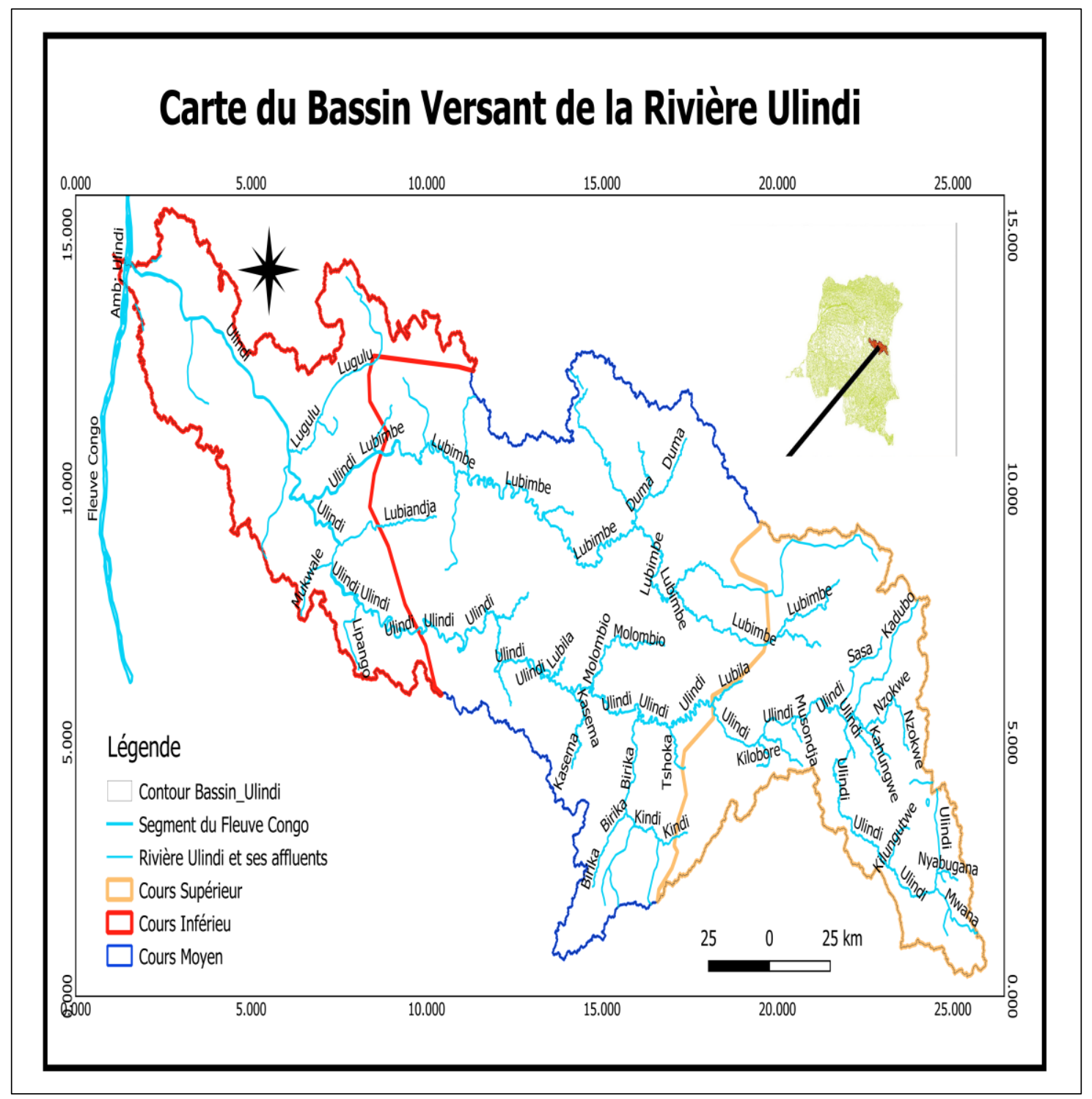

Figure 1 : Carte de localisation des études ichtyologiques réalisées dans le bassin de la rivière Ulindi, Est de la RD Congo. 


\section{MATÉRIEL ET MÉTHODES}

Dans cette revue, nous mettons l'accent sur les études axées sur la taxonomique des poissons du bassin supérieur du fleuve Congo (Lualaba) en se basant plus sur celles réalisées dans le sous-bassin de la rivière Ulindi depuis l'époque coloniale jusqu'en 2019.

Pour des raisons pratiques nous avons subdivisé le bassin de la rivière Ulindi en trois sous-bassins tenant compte de la zonation de Huet (1949) et de la couverture forestière basée sur l'altitude décroissante. Le cours supérieur correspond à la région de hautes altitude et occupe les territoires de Walungu et Mwenga). Le cours moyen correspond à la région de plaine (Territoire de Shabunda) et le Cours inférieur, c'est la partie embouchure (Territoire de Kalima et Punia). Les rivières déjà étudiées du bassin de 1'Ulindi pour lesquelles une liste d'espèces est fournie dans ce travail, appartiennent à deux groupes écologiques : une série d'affluents coulant en forêt (dont les rivières : Lugulu, Lubimbe, Nzokwe, Nzombe, Mwana, Emia et Kilobore) et une autre série en savane d'altitude parmi lesquelles les rivières (Gasharara, Kadubu, Kariagi, Ngombo, Nyabugana, Nalwegwe, Kahungwe, Sasa et Musondja).

Les nombreuses rivières mentionnées ne sont pas tout à fait limitées dans la région, par conséquent, la liste présentée ici est basée sur les fonds des collections de poissons vérifiées et logés dans le Musée Royal de l'Afrique Centrale de Tervuren, Belgique. Les informations complémentaires de la littérature (David et Poll, 1937; Marlier, 1954; Teugels et Thys, 1983; Lévêque et al., 1990; Teugels, 1996) ainsi que des avoirs d'autres musées par FishBase (Froese et Pauly, 2019) ainsi que des avoirs d'autres musées en ligne ont été également inclus (Koffelat, Munich, et Suffer 1988). L'orthographe du genre et les noms des espèces, les auteurs ainsi que les dates de publication originales ont été vérifiées dans les sources originales (Van Der Laan et al., 2014; Fricke et al., 2019; Froese et Pauly, 2019).

Les logiciels GRASS GIS, QGIS et Google Earth sous Windows 10 ont été respectivement utilisés pour la délimitation, la localisation et la production de la carte du bassin versant de la Rivière Ulindi tandis que pour l'organisation des références bibliographiques, le logiciel Zotero a été utilisé et le Microsoft Word 2016 nous a permis de faire la saisie ce texte.

\section{RÉSULTATS \\ Composition spécifique des poissons du la Rivière Ulindi}

L'analyse de toutes les sources bibliographiques à notre disposition (Pellegrin, 1935; David et Poll, 1937; Marlier, 1954; Marlier, 1958; Banister et Bailey, 1979; Koffelat,et al., 1988; Froese et Pauly, 2019) nous a permis de constater que le bassin de la Rivière Ulindi déjà étudié jusqu'à ce jour compte au total : quatre (4) ordres, neuf (9) familles, dix-sept (17) genres et 31 espèces de poissons (Tableau 1).

\section{Abondance relative des ordres et familles}

$\mathrm{La}$ structure non exhaustive du peuplement de l'ichtyofaune du bassin de la rivière Ulindi actuellement connue montre que les poissons sont représentés par l'ordre des Siluriformes $(51,6 \%)$ suivi des Perciformes avec 25,8\%, puis des Cypriniformes $(19,4 \%)$ et enfin viennent les Osteoglossiformes $(3,2 \%)$ (Figure 2).

La figure 3 révèle que la famille Mochokidae est plus représentés avec 19,4\% d'espèces de poissons déjà connues dans le bassin de la rivière Ulindi suivies des familles Amphilidae, Clariidae, et Cyprinidae avec $16,1 \%$ d'espèces chacune. La famille Cichlidae (13\%) suivis des Anabantidae (9,7\%) enfin, les Mastacembelidae, Distichodontidae et Mormyridae suivent avec 3,2\% chacune.

\section{Répartition des poissons par rivières} étudiées dans le Bassin de la rivière Ulindi

Les rivières déjà étudiées du bassin de 1'Ulindi appartiennent à deux groupes écologiques : une série d'affluents coulant en forêt, une autre série en savane d'altitude (Figure 4) et la répartition des espèces par rivière étudiées est présentée par le Tableau 2.

L'examen de la Figure 4 montre que beaucoup de sites dans ce bassin hydrographique n'ont pas encore été 
prospectés et nécessiteront des prospections ichtyologiques complémentaires dont les nôtres en cours.

Les données du Tableau 2, montrent que les espèces de poissons Amphilius kivuensis,
Clarias liocephalus, Opsaridium sp et Amphilius uranoscopus sont largement rependues dans les rivières déjà étudiées du bassin de la rivière Ulindi.

Tableau 1 : Liste systématique des poissons déjà connus dans le bassin de Ulindi.

\begin{tabular}{|c|c|c|c|}
\hline Ordres & Familles & Genres & Espèces \\
\hline \multirow{15}{*}{ Siluriformes } & \multirow{5}{*}{ Amphilidae } & \multirow{4}{*}{ Amphilius } & Amphilius kivuensis Pellegrin, 1933 \\
\hline & & & Amphilius sp. (Günther, 1864) \\
\hline & & & Amphilius jacksonii Boulenger, 1912 \\
\hline & & & Amphilus uranoscopus (Pfetter, 1889) \\
\hline & & Phractura & Phractura lindica Boulenger, 1902 \\
\hline & \multirow{5}{*}{ Clariidae } & \multirow{4}{*}{ Clarias } & Clarias liocephalus Boulenger, 1898 \\
\hline & & & Clarias camerunensis Lönnberg, 1895 \\
\hline & & & Clarias hilli Fowler, 1936 \\
\hline & & & Clarias jaensis Boulenger, 1909 \\
\hline & & Clariallabes & Clariallabes longicauda (Boulenger, 1902) \\
\hline & \multirow{5}{*}{ Mochokidae } & \multirow{5}{*}{ Chiloglanis } & Chiloglanis batesii Boulenger, 1904 \\
\hline & & & Chiloglanis marlieri Poll, 1952 \\
\hline & & & Chiloglanis micropogon Poll, 1952 \\
\hline & & & Chiloglanis reticulatus Roberts, 1989 \\
\hline & & & Chiloglanis pojeri Poll, 1944 \\
\hline \multirow{9}{*}{ Perciformes } & \multirow{3}{*}{ Anabantidae } & Ctenopoma & Ctenopoma multispine Peters, 1844 \\
\hline & & \multirow{2}{*}{ Microctenopoma } & Microctenopoma nanum (Günther, 1986) \\
\hline & & & Microctenopoma sp Norris, 1995 \\
\hline & \multirow{4}{*}{ Cichlidae } & Astatotilapia & Astatotilapia burtoni (Günther, 1894) \\
\hline & & Chromodotilapia & $\begin{array}{l}\text { Chromidotilapia schoutedeni (Poll, Thys, van, } \\
\text { den \& Audenaerde, 1967) }\end{array}$ \\
\hline & & \multirow{2}{*}{ Oreochromis } & Oreochromis niloticus (Linnaeus, 1758) \\
\hline & & & Oreochromis spilurus (Günther, 1894) \\
\hline & \multirow{3}{*}{ Mastacembelidae } & \multirow{3}{*}{ Mastacembelus } & Mastacembelus frenatus Boulenger, 1901 \\
\hline & & & Mastacembelus congicus Boulenger, 1896 \\
\hline \multirow{6}{*}{ Cypriniformes } & & & Labeobarbus somereni (Boulenger, 1911) \\
\hline & \multirow{4}{*}{ Cyprinidae } & Labeobarbus & $\begin{array}{l}\text { Labeobarbus pellegrini (Bertin \& Estève, } \\
\text { 1948) }\end{array}$ \\
\hline & & Raiamas & Raiamas moorii (Boulenger, 1900) \\
\hline & & Barbus & Barbus sp Cuvier \& Cloquet,1816 \\
\hline & & Opsarium & Opsaridium ubangiense (Pellegrin, 1901) \\
\hline & Distichodontidae & Mesoborus & Mesoborus crocodilus Pellegrin, 1900 \\
\hline Osteoglossiformes & Mormyridae & Mormyrus & Mormyrus caballus Boulenger, 1898 \\
\hline
\end{tabular}




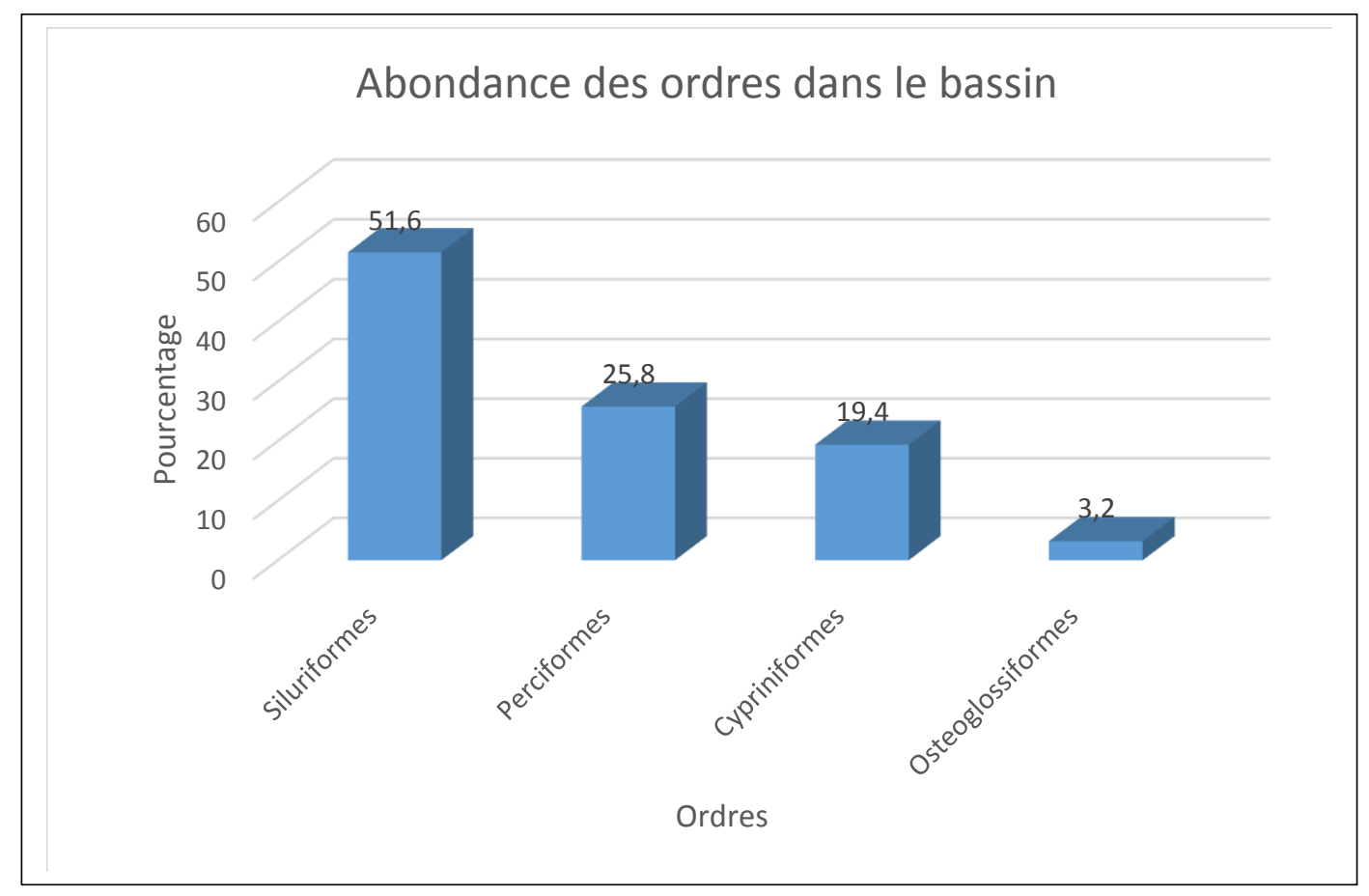

Figure 2: Abondance relative des ordres.

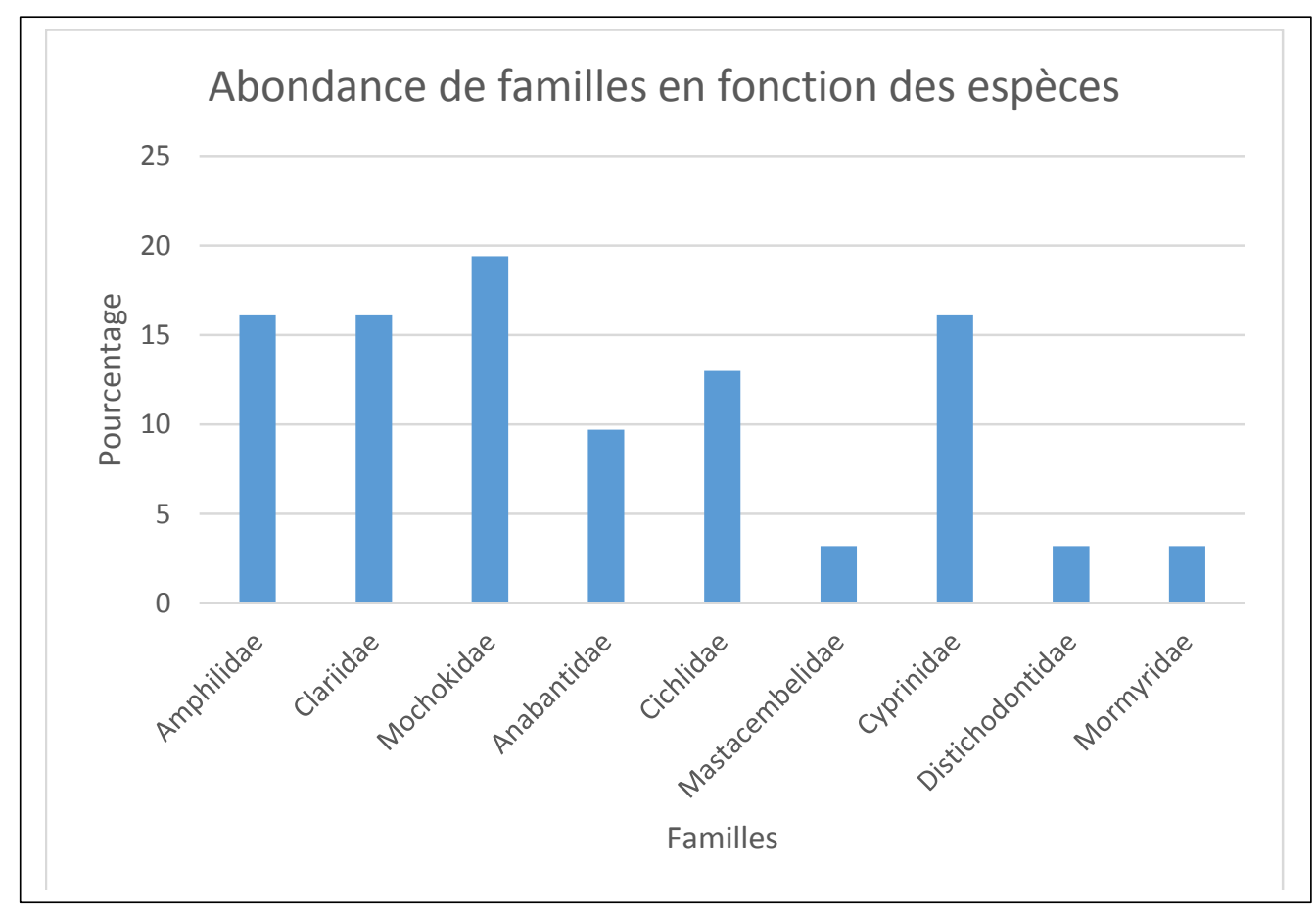

Figure 3 : Abondance des familles en fonction des espèces. 


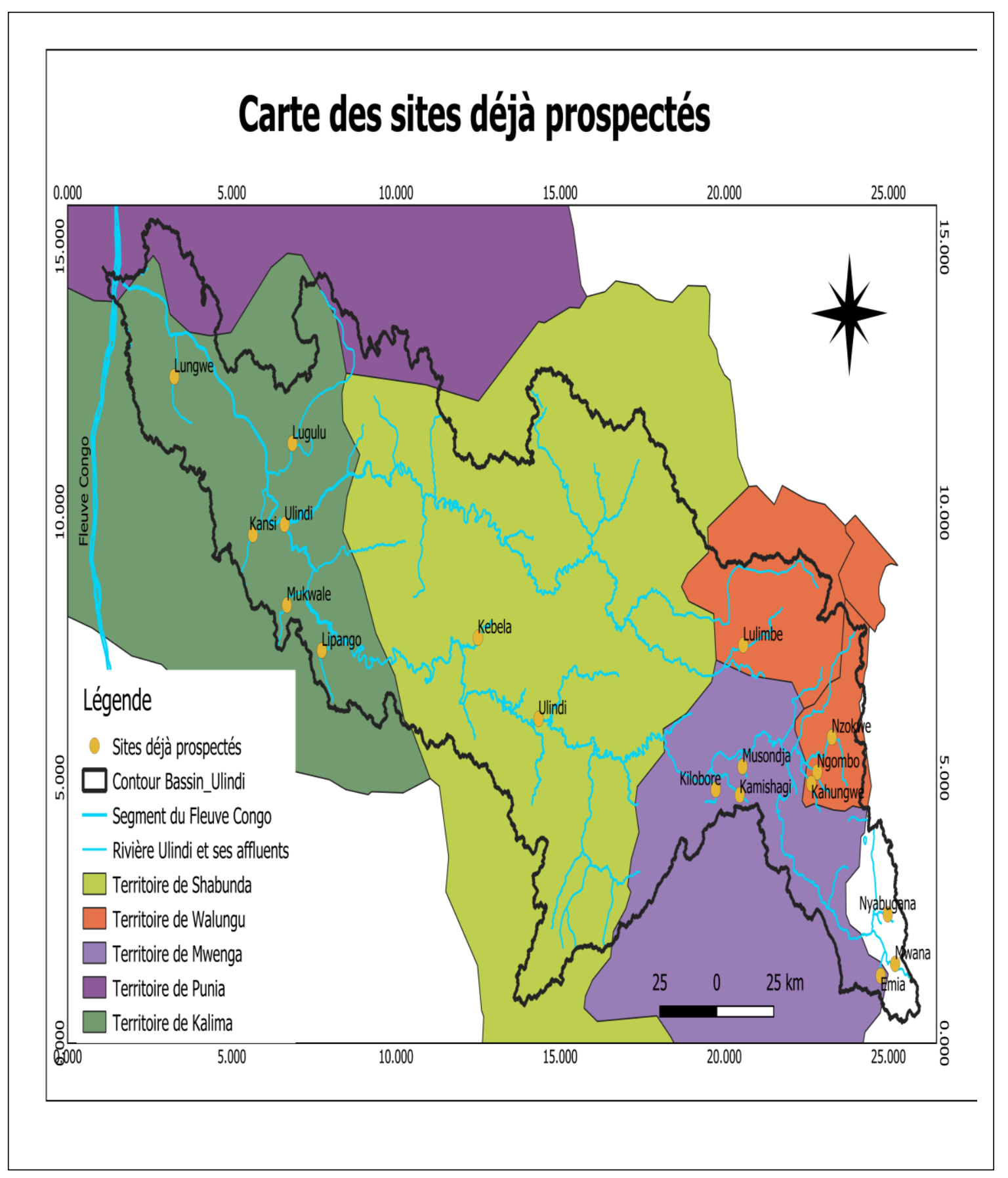

Figure 4 : Carte de localisation des études ichtyologiques déjà réalisées dans le bassin de la rivière Ulindi, Est de la RD Congo. 
Tableau 2 : Répartition des différentes espèces de poissons du Bassin de la rivière Ulindi actuellement connues.

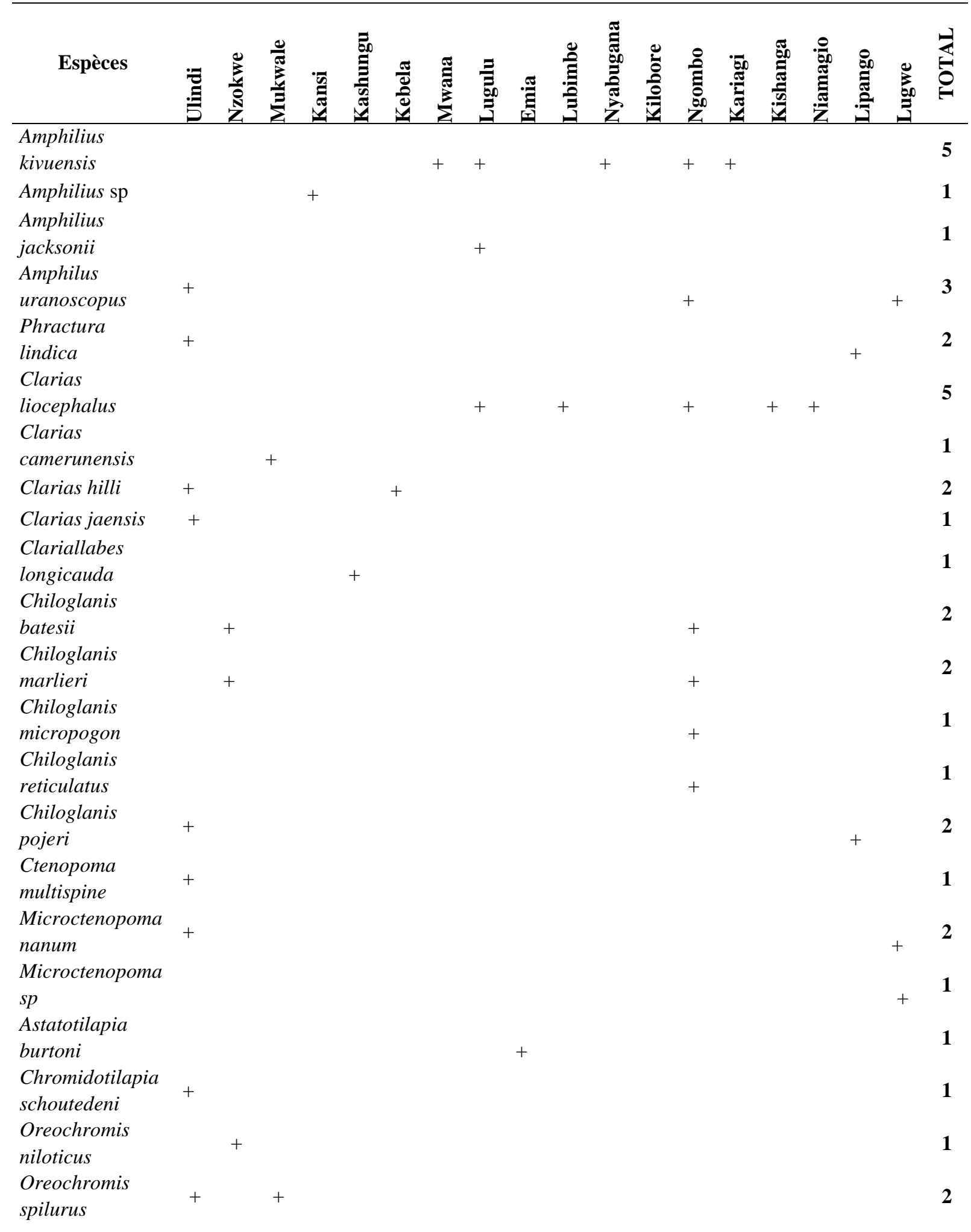




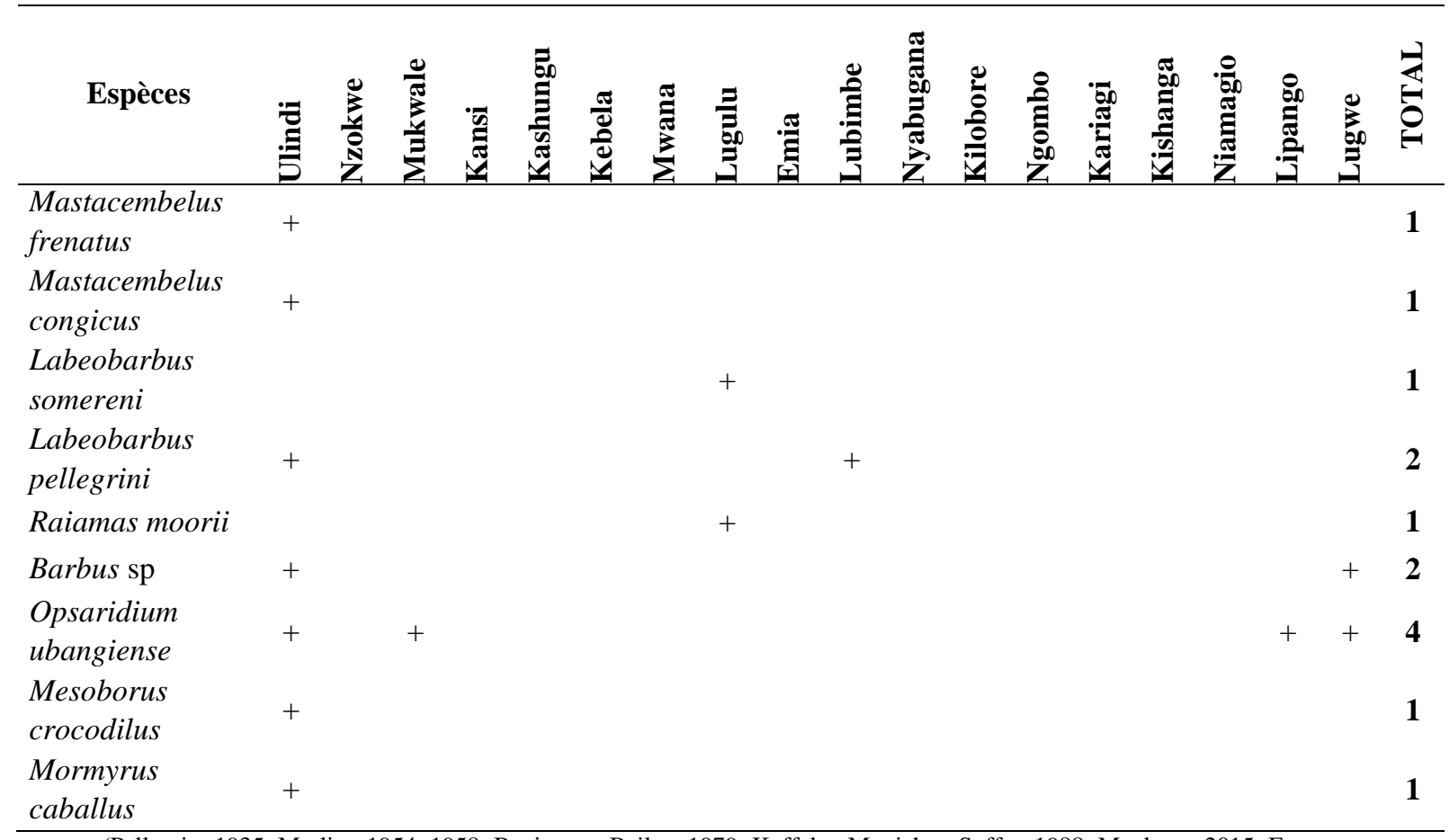

(Pellegrin, 1935; Marlier, 1954, 1958; Banister et Bailey, 1979; Koffelat, Munich et Suffer, 1988; Moelants, 2015; Froese et Pauly, 2019).

\section{DISCUSSION}

La richesse spécifique des quelques rivières déjà étudiées dans le bassin de la rivière Ulindi telle que relevée par la littérature est de 31 espèces réparties dans 17 genres, 9 familles et 4 ordres. En effet, elle reste la valeur de référence pour les études ultérieures dans cette partie du bassin du Congo.

Cependant, Kisekelwa (2014) ayant travaillé sur le cours moyen de la rivière Lowa dans la même partie du bassin du Congo en Territoire de Walikale, avait trouvé que les poissons de la rivière Lowa comprenaient 19 espèces. En outre, Bulimwengu (2009), quant à lui, avait travaillé dans la même partie du bassin du Congo en province Orientale dans la réserve d'Okapis. Il avait trouvé que les poissons des rivières Epulu comprenaient 46 espèces, Nduye: 39 espèces, Epene: 33 espèces tandis que les sites Edoro et Lelo avaient respectivement 20 et 16 espèces chacun alors que le site Esaye n'a que 20 espèces. Ces deux résultats montrent bien une richesse spécifique plus élevée que celle déjà connues de quelques sites du bassin de la rivière Ulindi. En effet, lors de travaux de terrain, ces chercheurs cihaut cités ont parcouru l'ensemble des bassins versants des rivières Epulu, Nduye, Epene, Edoro, Lelo et Esaye. C'est ce qui a conduit à une bonne idée de leur diversité ichtyologique. Par ailleurs, ils ont également travaillé dans de petites rivières de cette partie, ce qui permet d'améliorer et de compléter l'inventaire de cette faune ichtyologique, ce qui n'est pas encore le cas pour le bassin de la rivière Ulindi.

$\mathrm{Au}$ point de vue abondance relative, dans la partie prospectée du bassin de la rivière Ulindi, les Ordres sont dominés par les Siluriformes (51,6\%) et Perciformes (25,8\%) et les Cypriniformes $(19,4 \%)$ ce qui n'est pas étonnant car ils sont parmi les ordres largement dominants en Afrique (Lowe Mc Connell,1987). Ce résultat s'écarte de ce qu'avait trouvé Mbega (2003) dans le bassin inférieur de l'Ogooué. Dans ce bassin, les Perciformes $23,7 \%$, les Siluriformes représentaient 19,4\%, les Characiformes $14,4 \%$ et les Mormiformes $10,1 \%$. Il y a donc une quasi différence dans la dominance des ordres entre cette partie du bassin de la rivière 
Ulindi et le bassin inférieur de l'Ogooué. La dominance des Perciformes dans le bassin inférieur de l'Ogooué, serait due à la présence des lacs dans le bassin de l'Ogooué. Les Perciformes se développent mieux dans les milieux lentiques.

Par ailleurs, Mbalassa (2008) ayant travaillé dans la rivière Ishasha a relevé la dominance de Perciformes avec 44\%, suivie de celle des Cypriniformes avec 26\%, après viennent celles des Siluriformes, Mormyriformes qui étaient respectivement de $13 \%$ et $7 \%$. On se rend compte, dans la région intertropicale que les Perciformes, les Osteoglossiformes, les Siluriformes, les Cypriniformes semblent être des ordres beaucoup plus importants. Les différences remarquées entre nos résultats et ceux de Mbalassa (2008) sont dues à la saisonnalité de l'échantillonnage. En plus, les sites d'échantillonnage de Mbalassa (2008) étaient proches de l'embouchure de la rivière Ishasha dans le lac Victoria, ce qui traduit des migrations longitudinales des poissons du lac Victoria et qui ont été pêchés dans cette partie de la rivière Ishasha. Cette hypothèse est aussi confirmée par Mbega (2003) qui a trouvé que les sites proches des océans ou des lacs, sont peuplés par des poissons venus de ces grands écosystèmes et ayant migré vers ces sites. En plus, la partie de la Ulindi concernée dans le cadre de ce travail est caractérisée par des chutes qui limiteraient la montée des poissons d'aval vers l'amont (Mbega, 2003 ; Mbalassa, 2008). Ces différents facteurs hydromorphologiques limiteraient davantage la richesse ichtyologique en amont de la rivière Ulindi.

En outre, Mbega (2003), Mbalassa (2008) et Kisekelwa (2014) affirment que les Mormyriformes, Siluriformes, Cypriniformes et les Characiformes sont parmi des ordres les plus riches en espèces et les plus répandus au monde. La présence des ordres Siluriformes, Cypriniformes dans la rivière Ulindi pourrait être expliquée, non seulement par leur potentiel d'adaptation mais aussi par leur capacité de vivre dans une variété d'habitats en supportant même des conditions environnementales difficiles. Ils sont également caractéristiques des rivières tropicales (Lowe Mc Connell,1987). Comparativement aux observations faites par Mbalassa (2008) dans la rivière Ishasha, les ordres et familles trouvés dans l'Ulindi Supérieure sont aussi rencontrés dans d'autres rivières tropicales, spécialement dans les rivières sub-sahariennes.

S'agissant des familles des poissons des rivières prospectées dans le bassin de la rivière Ulindi, elles figurent parmi les familles ayant une large distribution en Afrique (Greenwood, 1983). Il s'agit des familles de Clariidae, Cichlidae et Cyprinidae. Elles sont pour la plupart répertoriées dans les écosystèmes de l'Afrique de l'Ouest (Lévèque et al., 1990). Les familles Mochokidae, Cyprinidae, Clariidae, Amphilidae et Cichlidae sont dominantes dans les sites prospectés du bassin de la rivière Ulindi. Cependant, la faible abondance des familles des Anabantidae, Mormyridae et Mastacembelidae Distichodontidae serait due probablement aux chutes qui séparent l'aval et la partie concernée par la plupart de ces études.

La plupart de ces rivières étudiées sont des rivières de montagne et appartiennent au cours supérieur de la Ulindi à l'exception des rivières Kebela, Lipango, Lugulu et Lungwe qui sont du cours moyen et inférieur de la rivière Ulindi. Ces biotopes sont difficiles à exploiter par des pêcheurs à cause de la vitesse du courant des eaux de cette rivière.

La plus grande partie de l'Ulindi moyenne et inférieure occupe la forêt du Maniema et de toutes ces études antérieures pourtant, elle représente un milieu bien distinct des cours d'eau de montagne. Ceci signifie que le bassin de la rivière Ulindi nécessite encore d'autres études d'inventaires systématiques des poissons sur tout son parcours supérieur (Ulindi et ses affluents non montagneux), le reste de son cours moyen et l'ensemble du cours inférieur. Ces prospections, une fois réalisées, permettront probablement d'identifier d'autres espèces non encore connues.

Les résultats de cette recherche documentaire constituent non seulement un point de départ pour des études ultérieures sur la diversité ichtyologique dans le Bassin de la rivière Ulindi, mais ils sont aussi, pour les 
autorités administratives ayant la gestion de l'environnement dans leurs attributions, une base des données importantes pouvant leur être utile dans la gestion de la biodiversité ichtyologique de cet écosystème à intérêt socio-économique considérable dans la région.

\section{Conclusion}

La liste systématique des poissons d'Ulindi présentée dans cette revue est le résultat de la méta-analyse des différentes études taxonomiques sur l'ichtyofaune du Bassin de la Rivière Ulindi réalisées depuis l'époque coloniale du Congo-Belge jusqu'en 2019. Cette étude avait pour objectif d'identifier et d'analyser les études disponibles sur les poissons du bassin de la rivière Ulindi, affluent du fleuve Congo dans son bassin supérieur (Lualaba) dans le but de : 1) produire une liste systématique de toutes les espèces de poissons déjà connues dans ce bassin hydrographique, leur distribution par sites prospectés ; 2) identifier les différentes lacunes pouvant susciter des recherches futures. En considérant la très faible étendue prospectée et les matériels de pêche trop limité qui ont été utilisés, on peut penser que ce bassin renferme encore des espèces inconnues. Au moment où on assiste à une prolifération des dragues pour les extractions minières en utilisant le mercure tout au long de cet important affluent du Fleuve Congo, il est souhaitable d'intensifier les recherches ichtyologiques afin que les espèces probablement nouvelles ne disparaissent avant qu'elles ne soient connues. De manière générale, une meilleure connaissance de la biodiversité ichtyologique des rivières du bassin du Congo et de la Rivière Ulindi en particulier permettra une meilleure gestion de celle-ci d'une part et constituera d'autre part une bonne base pour définir de programme de surveillance de nos milieux aquatiques. Ainsi, dans l'état actuel des connaissances sur l'ichtyofaune du Bassin de la Rivière Ulindi, deux axes de recherche s'avèrent intéressant à développer : d'une part, une étude approfondie de la taxonomie et de la systématique des cours supérieur, moyen et inférieur et d'autre part, une évaluation d'impacts des activités minières en pleine croissance le long de la rivière Ulindi, sur la nature de cet écosystème ainsi que la physiologie de son ichtyofaune.

\section{CONFLIT D'INTERETS}

Les auteurs déclarent qu'il n'y a aucun conflit d'intérêt.

\section{CONTRIBUTIONS DES AUTEURS}

GMO est intervenu dans toutes les phases de l'étude. Il s'agit de la conception de l'étude, la collecte des données, le dépouillement, le traitement et l'analyse des données et la rédaction du présent manuscrit. VNM, JCM, GN et CS sont intervenus dans la conception de l'étude et la correction du manuscrit. AS est intervenu dans la délimitation et la cartographie du bassin versant. JMM, PLD et LHM sont intervenus dans le traitement des données et dans la correction du manuscrit.

\section{REMERCIEMENTS}

Nous remercions le Professeur Dr. Jos Snoeks et toute l'équipe du l'Ichtyologie du Musée Royal de l'Afrique Centrale de Tervuren en Belgique et particulièrement $\mathrm{Mr}$. Tobias MUSSCHOOT qui ont mis à notre disposition une base des données et auprès de qui une grande partie de la documentation (sources primaires) a été obtenue. Nous remercions également les autorités de l'ARESCCD, et de l'Ecole Doctorale de l'Université du Burundi et à travers elles les autorités de la DGCD pour avoir financé le séjour de GMO en Belgique.

\section{RÉFÉRENCES}

Banister KE, Bailey RG. 1979. Fishes Collected by the Zaire River Expedition, 1974-75. Zoological Journal of the Linnean Society, 66(3): 205-49. DOI: http://doi.org/10.1111/j.10963642.1979.t b01909.x.

Danadu MC, Vreven E, Moelants T, Ulyel AP, Snoeks J. 2016. Two Case Studies on $<$ EM $>$ Synodontis</EM > Cuvier, 1816 (Siluriformes: Mochokidae) from the Congo Basin (DRC) . Fifth International Conference of the Pan African Fish and 
Fisheries Association (PAFFA5). http://orfeo.kbr.be/handle/internal/1805.

Decru E, Vreven E, De Gelas K, Verheyen E, Snoeks J. 2017. Species Richness in the African Pike Genus Hepsetus: A Perfect Match between Genetics and Morphology: Genetics and Morphology of Hepsetus. Journal of Fish Biology, 91 (2): 617-27. DOI: https://doi.org/10.1111/jfb.13376.

Decru E, Vreven E, Danadu C, Walanga A, Mambo T, Snoeks J. 2017. Ichthyofauna of the Itimbiri, Aruwimi, and Lindi/Tshopo Rivers (Congo Basin): Diversity and Distribution Patterns ». Acta Ichthyologica et Piscatoria, 47(3): 225- 247.

DOI: https://doi.org/10.3750/AIEP/02085.

Harrison IJ, Brummett R, Melanie L, Stiassny J. 2016. The Congo River Basin. In The Wetland Book, Finlayson MC, Milton RG, Prentice R C, Davidson NC (ed). Dordrecht: Springer Netherlands; 1- 18. DOI: https://doi.org/10.1007/978-94007-6173-5_92-1.

Hermoso V, Abell R, Linke S, Boon P. 2016. The Role of Protected Areas for Freshwater Biodiversity Conservation: Challenges and Opportunities in a Rapidly Changing World: Freshwater Protected Areas ». Aquatic Conservation: Marine and Freshwater Ecosystems, 26: 3- 11. DOI: https://doi.org/10.1002/aqc.2681.

Ibala A, Masmoudi A. 2010. Accounting for the Armature Magnetic Reaction and Saturation Effects in the Reluctance Model of a New Concept of Claw-Pole Alternator . IEEE Transactions on Magnetics, 46(11): 3955-3961. DOI: https://doi.org/10.1109/TMAG.2010.205 5882.

Kisekelwa T, Hyangya L, Masilya MP, Isumbisho M, Kaningini M. 2014. Contribution à l'inventaire systématique des poissons de la Rivière lowa en territoire de Walikale. Cahiers $d u$ CERUKI, (Numéro Spécial Alphonse Byamungu): 20-28.
Kisekelwa T, Snoeks J, Nshombo M, Isumbisho M, Vreven E. 2016. Towards a Checklist of the Fishes of Kahuzi-Biega National Park and Its Surroundings, Eastern Congo River Basin (DRC) ». Fifth International Conference of the Pan African Fish and Fisheries Association (PAFFA5). https://orfeo.kbr.be/handle/internal/1807.

Koffelat Maurice, Munich, Ernest Suffer. 1988. Catalogue des types des poissons du Musée d'Histoire Naturelle de Bâle (Naturhistorisches

Museum Basels). Verhandl. Naturf. Ges. Bast., 98: 51- 57.

Marlier G. 1954. Recherches hydrobiologiques dans les rivières du Congo oriental: Etude écologique. Hydrobiologia, 6(3-4): 225- 264.

DOI: https://doi.org/10.1007/BF00053675.

Marlier G. 1958. Recherches hydrobiologiques dans les rivières du Congo Oriental. Composition des Eaux. 1) La Conductibilité électrique. Ann. Soc. $R$. Zool. Belg., 84(1): 217- 227.

Musschoot T, Snoeks J. 2017. ReEstablishment of the Genus Monostichodus Vaillant 1886 (Characiformes, Distichodontidae). Journal of Fish Biology, 90(3): 1080-1082. DOI: https://doi.org/10.1111/jfb.13218.

Mukabo OG, Cikwanine KDD, Micha JC, Nshombo MV, Rwakana RO, Bizuru NP, Muhirwa BG, Mboboci ESD, Mudimbe KP. 2017. Ecologie alimentaire de Labeobarbus altianalis (Boulenger, 1900) du Bassin de la rivière Luhoho, en territoire de Kalehe (Sud-Kivu, R.D. Congo). International Journal of Biological and Chemical Sciences, 11(1): 208-231.

DOI: https://doi.org/10.4314/ijbcs.v11i1.17.

Pellegrin J. 1935. Poissons de la région du Kivu récoltés par M. Guy Babault. Revue de Zoologie et de Botanique Africaines, 27(3): 376- 385.

Teugels GG. 1996. Taxonomy, Phylogeny and Biogeographie of Catfishes 
(Ostariophysi, Siluroidei) : An Overview. Aquatic Living Resources, 9: 9- 34.

Van Der Laan R, Eschmeyer WN, Fricke R. 2014. Family-Group Names of Recent Fishes. Zootaxa, 3882(1): 1. DOI: https://doi.org/10.11646/zootaxa.3882.1. 1.

Wamuini LS, Vreven E, Vandewalle P, Mutambue S, Snoeks J. 2010. Contribution à la connaissance de l'ichtyofaune de l'Inkisi au Bas-Congo (RD du Congo). Cybium, 34(1): 83-91.

COSOC-GL A. 2015. Vers une ruée d'or à Shabunda: Pratiques et Impacts de l'exploitation minière par dragues. Rapport de Recherche, Coallition de la cociété civile de la Région des Grands Lacs, Bukavu, RD Congo, p.1-41.

David L, Poll M. 1937. Contribution à la faune ichtyologique du Congo Belge. Annales du Musée du Congo-Belge, 3-5(1).

Freyho J, Khorozyan I, Fayvush G. 2015. Freshwater Ecosystems and Biodiversity: Atlas of Freshwater Key Biodiversity Areas in Armenia. Armenia: Yerevan. WWF-Armenia.

Goudiaby, Khady D, Ifan CAD. 2015. «Systématique des poissons », p. 45.
Fricke R, Eschmeyer WN, Van der Laan R. 2019. Catalogue des poissons d'eschmeyer: genera, espèces, Références. California Academy of Sciences.

http://researcharchive.calacademy.org/re search/ichthyology/catalog/fishcatmain.a sp).

Froese R, Pauly D. 2019. FishBase. http://www.fishbase.org/search.php.

Lévêque C, Paugy D, Teugels GG. 1990. Faune Des Poissons d'eaux Douces et Saumâtres d'Afrique de l'Ouest. Editions de l'ORSTOM, Collection Faune Tropicale (no: 28): Tervuren, Belgique.

Moelants T. 2015. Diversity and ecology of the ichthyofauna of the Middle and Upper Congo basin: a case-study in the region of the Wagenia falls (Democratic Republic of the Congo).

Teugels GG, Van den Audenaerde T. 1983. Systematische studie van de Afrikaanse Clarias-vissoorten (Piscess : Siluriformes, Clariidae). PhD Thesis, Katholieke Universiteit Leuven, Leuven, Belguim. 